Submitted version of: W. P. du Plessis, "Analysis of Path-Length Effects in Multi-Loop Cross-Eye Jamming," IEEE Transactions on Aerospace and Electronic Systems, vol. 53, no. 5, pp. 2266-2276, Oct. 2017. Published version is available online at: http://ieeexplore. ieee.org/document/7891642/

(C) 2017 IEEE. Personal use of this material is permitted. Permission from IEEE must be obtained for all other uses, in any current or future media, including reprinting/republishing this material for advertising or promotional purposes, creating new collective works, for resale or redistribution to servers or lists, or reuse of any copyrighted component of this work in other works.

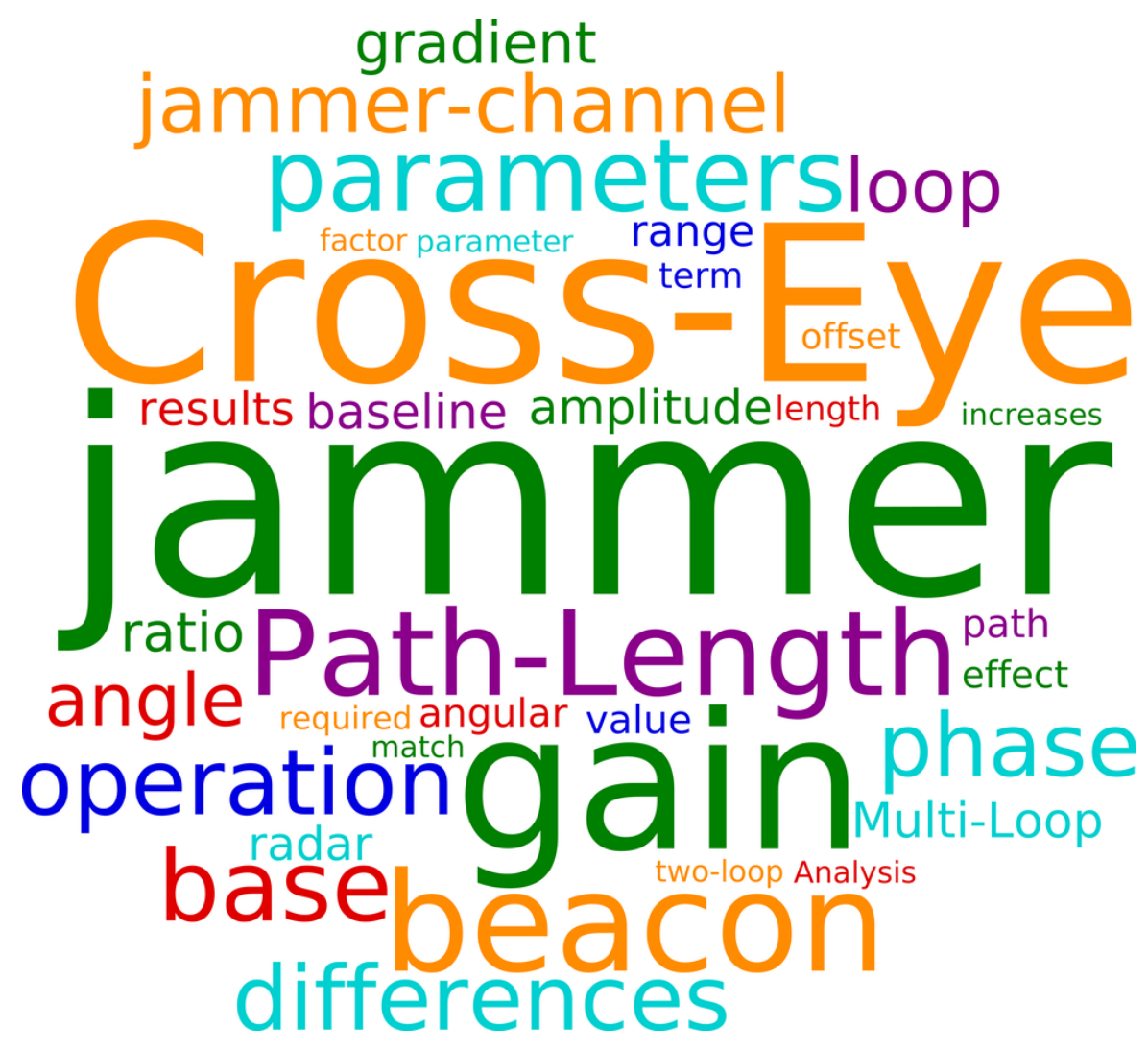

\title{
ABBREVIATIONS
}

CSIR Council for Scientific and Industrial Research

EA electronic attack

ECM electronic countermeasures

JSR jammer-to-signal ratio

LHS left-hand side

NRF National Research Foundation of South Africa

RHS right-hand side 


\section{Analysis of Path-Length Effects in Multi-Loop Cross-Eye Jamming}

W. P. du Plessis, Senior Member, IEEE

\begin{abstract}
The effect of path-length differences on multiloop retrodirective cross-eye jammers is evaluated. It is shown that such jammers may act as beacons, and the conditions under which this occurs are investigated for two-loop jammers. The sensitivity of the two-loop crosseye gain to path-length differences is also studied and is found to be small for small path-length differences, but to increase rapidly. The effect of the two-loop crosseye jammer parameters on path-length effects is also considered.
\end{abstract}

Index Terms-Cross-eye jamming, electronic warfare (EW), electronic countermeasures (ECM), and radar countermeasures.

Manuscript received 9 October 2015; revised 3 April 2016 and 4 January 2017. This work is based on the research supported in part by the National Research Foundation of South Africa (NRF) (Grant specific unique reference number (UID) 85845). The NRF Grantholder acknowledges that opinions, findings and conclusions or recommendations expressed in any publication generated by the NRF supported research are that of the author(s), and that the NRF accepts no liability whatsoever in this regard.

W. P. du Plessis is with the University of Pretoria, Pretoria, 0002, South Africa (e-mail: wduplessis@ieee.org).

Digital Object Identifier ??.????/???.2017.??????

\section{INTRODUCTION}

Cross-eye jamming is an electronic attack (EA) (also known as electronic countermeasures (ECM) technique which seeks to induce an angular error in a threat radar [1]-[10]. This goal is achieved by artificially recreating the worst angular error which can be caused by glint with the result that cross-eye jamming is occasionally referred to as artificial glint. Glint is a naturally-occurring phenomenon which affects all radars and can lead to extremely large angular errors [11], [12]. As a result of being based on a naturally-occurring phenomenon, crosseye jamming has a number of benefits over other EA techniques which seek to induce angular errors in radars [13].

Unfortunately, there are a number of challenges associated with the implementation of cross-eye jammers. The magnitude of these challenges is demonstrated by the fact that the first cross-eye jammer systems suitable for operational use on aircraft and ships were only publicly disclosed in 2000 [3], while patents describing cross-eye jamming were submitted in the late 1950s [1], [2]. These challenges are mainly related to the extremely high jammer-to-signal ratio (JSR) which results from the signal cancellation inherent in cross-eye jamming [14], [15] and the extremely fine tolerances required to achieve large angular errors [16].

The simultaneous use of multiple cross-eye jamming systems operating together (multi-loop cross-eye jamming) has been proposed as a means to overcome these challenges [17]-[22]. The JSR required from each jammer system is reduced by the simultaneous use of more than one jammer loop. Furthermore, the additional degrees of freedom offered by multiple cross-eye jammer loops means that tolerance requirements can be loosened, and larger angular errors and/or increased angular coverage can be achieved.

However, it has recently been demonstrated that the performance of multi-loop cross-eye jammers can be compromised by differences between the path lengths of the signals through each jammer loop [23]. Worryingly, it was shown that the angular error induced in the threat radar may be negligible, thereby providing a powerful radar return at the position of the jammer, and in this way, actually assisting the radar in tracking the platform on which the jammer is mounted (beacon operation). It would be preferable not to use a cross-eye jammer if beacon operation is possible because the jammer could actually increase the threat posed by the radar.

This difficulty does not arise in single-loop cross-eye 
jammers as a result of the retrodirective implementation. The retrodirective implementation ensures that the path lengths of the two signals through the jammer are identical by retransmitting the signal received by each jammer antenna from the other antenna. So important is the retrodirective implementation that at least one author restricts the term cross-eye jamming to systems which use the retrodirective implementation [7].

The results presented previously use Monte-Carlo simulations to demonstrate both the existence and magnitude of the problems which can be caused by path-length differences [23]. However, this approach does not provide insight into the underlying causes of these problems or how to address them.

To overcome this limitation, a rigorous analysis of path-length effects in multi-loop cross-eye jamming is provided in Section II. The large number of parameters complicates the analysis to the point that obtaining insight into the underlying effects becomes impossible, so only the two-loop case is studied in detail. The conditions which can lead to beacon operation are considered in Section III as a cross-eye jammer which operates as a beacon is more dangerous than not having a jammer. The sensitivity of the angular error to pathlength differences is then investigated in Section IV to determine how the effect of path-length differences can be minimised. System performance is considered in Section $\mathrm{V}$ to illustrate how the presented analysis can be applied to cross-eye jammer design, and significant improvements over previous results are demonstrated. Finally, the main results are summarised in Section VI

\section{ANALYSIS}

The mathematical analysis underpinning the evaluation of the effect of path-length differences in Sections III, IV and V is provided below. Section II-A briefly summarises the relevant previously-published material, and Section II-B extends this analysis to consider the effect of path-length differences on a two-loop cross-eye jammer in detail.

While the analysis below only explicitly considers phase-comparison monopulse radar, it has been shown that the results are applicable to any monopulse radar [10], [24].

\section{A. Multi-Loop Retrodirective Cross-Eye Jamming}

A summary of the analysis of multi-loop cross-eye jamming in [22] is provided below.

Fig. 1 shows the geometry of an engagement involving a monopulse threat radar on the left and a two-loop crosseye jammer on the right. The conceptual operation of the jammer loops is shown in Fig. 2

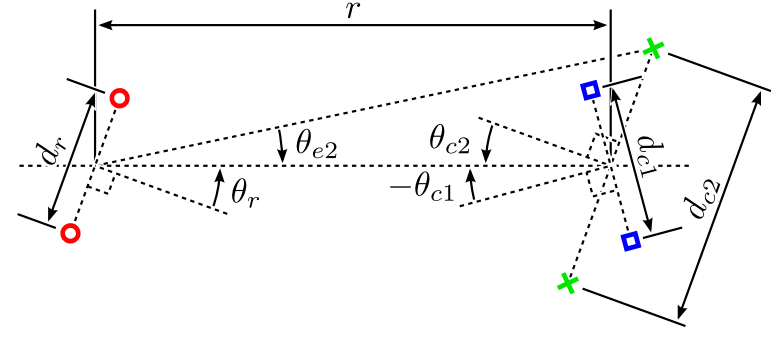

Fig. 1. The geometry of a multi-loop cross-eye jamming scenario [22], [23]. The phase centres of the antenna elements comprising the phase-comparison monopulse radar, and the inner and outer jammer loops are represented by circles, squares, and crosses respectively.

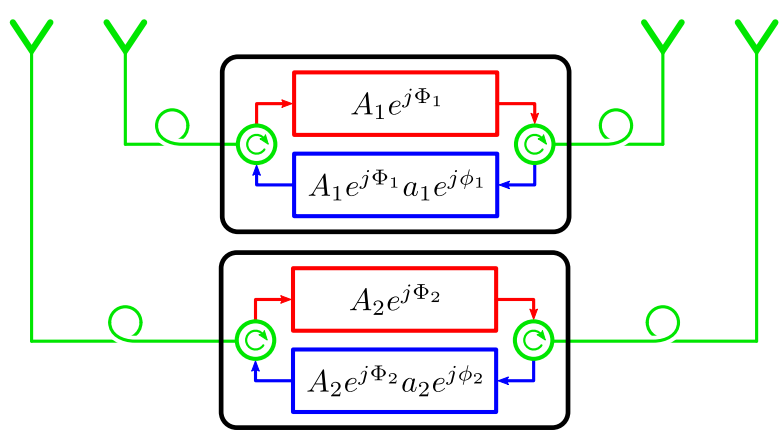

Fig. 2. The implementation of two retrodirective cross-eye jammer loops showing the meanings of $a_{n}, \phi_{n}, A_{n}$, and $\Phi_{n}$.

The parameters listed below will be used throughout this work [22].

- $\beta$ is the free-space phase constant given by

$$
\beta=\frac{2 \pi}{\lambda}
$$

where $\lambda$ is the wavelength.

- $r$ is the range from the radar to the centre of the jammer, as shown in Fig. 1 .

- $d_{r}$ is the separation of the radar antenna-element phase centres, as shown in Fig. 1.

- $\theta_{r}$ is the angle from the radar's boresight direction to the centre of the jammer, as shown in Fig. 1

- $d_{c n}$ is the antenna spacing for jammer loop $n$ (the jammer baseline), as shown in Fig. 1 .

- $\theta_{c n}$ is the rotation of the $n^{\text {th }}$ jammer loop's broadside direction from the radar, as shown in Fig. 1 .

- $\theta_{e n}$ is half the angular separation of the $n^{\text {th }}$ jammer loop's antennas from the radar's perspective, as shown in Fig. 1, and is given by

$$
\theta_{e n} \approx \frac{d_{c n}}{2 r} \cos \left(\theta_{c n}\right) \text {, }
$$

which is very accurate as long as $r \gg d_{c n}[10]$.

- $a_{n}$ and $\phi_{n}$ are the jammer-channel parameters of jammer loop $n$, as shown in Fig. 2 .

- $A_{n}$ and $\Phi_{n}$ are the jammer-loop parameters of jammer loop $n$ as a whole, as shown in Fig. 2. 
The antenna parameters can summarised by [22]

$$
\begin{aligned}
P_{a n}\left(\theta_{r}, \theta_{c n}, \theta_{e n}\right) & \\
= & P_{r}\left(\theta_{r}-\theta_{e n}\right) P_{r}\left(\theta_{r}+\theta_{e n}\right) \times \\
& P_{c n}\left(\theta_{c n}-\theta_{e n}\right) P_{c n}\left(\theta_{c n}+\theta_{e n}\right) \\
\approx & {\left[P_{r}\left(\theta_{r}\right) P_{c n}\left(\theta_{c n}\right)\right]^{2} }
\end{aligned}
$$

where $P_{r}(\theta)$ is the pattern of the antenna elements comprising the phase-comparison monopulse radar antenna, and $P_{c n}(\theta)$ is the pattern of the antennas of the $n^{\text {th }}$ jammer loop. The approximation in (4) is accurate when the radar antenna parameters are predominantly determined by the spacing of the antenna elements $\left(d_{r}\right)$ giving $P_{r}\left(\theta_{r}-\theta_{e n}\right) \approx P_{r}\left(\theta_{r}\right) \approx P_{r}\left(\theta_{r}+\theta_{e n}\right)$, and when the jammer antennas either have broad beamwidths or are phased arrays which steer their beams towards the threat radar giving $P_{c n}\left(\theta_{c n}-\theta_{e n}\right) \approx P_{c n}\left(\theta_{c n}\right) \approx$ $P_{c n}\left(\theta_{c n}+\theta_{e n}\right)$. Equation 4 can be further simplified to

$$
P_{a n}\left(\theta_{r}, \theta_{c n}, \theta_{e n}\right) \approx\left[P_{r}\left(\theta_{r}\right) P_{c}\left(\theta_{c}\right)\right]^{2}
$$

by capturing remaining differences between the jammer antenna parameters in the jammer-loop parameters $\left(A_{n}\right.$ and $\left.\Phi_{n}\right)$ to allow $P_{c n}\left(\theta_{c n}\right)=P_{c}\left(\theta_{c}\right)$. The antenna factor in (5) is identical for all jammer loops, so the effects of the radar and jammer antenna element patterns cancel out and do not affect the result.

The following parameters are used to simplify the notation [9], [10]

$$
\begin{aligned}
k & \approx \beta \frac{d_{r}}{2} \sin \left(\theta_{r}\right) \\
k_{c n} & \approx \beta \frac{d_{r}}{2} \cos \left(\theta_{r}\right) \theta_{e n}
\end{aligned}
$$

where the approximations are extremely accurate for practical cross-eye jamming scenarios where the range is far greater than the jammer baseline $\left(r \gg d_{c n}\right)$ [10].

The angular error of a monopulse radar is computed from an intermediate parameter known as the monopulse ratio, which is computed from the ratio of the differencechannel return to the sum-channel return [12]. For a retrodirective cross-eye jammer, the monopulse ratio is given by [9], [22]

$$
\begin{aligned}
M_{M} & \approx \frac{\sin (2 k)+\sin \left(2 k_{c N}\right) G_{C N}}{\cos (2 k)+\cos \left(2 k_{c N}\right)} \\
& \approx \tan (k)+\frac{k_{c N}}{\cos ^{2}(k)} G_{C N}
\end{aligned}
$$

where $k_{c N}$ is the value of $k_{c n}$ for loop $N$ in the multiloop case, $G_{C N}$ is the cross-eye gain of $N$ loops, and the accurate approximations [25]

$$
\begin{gathered}
\cos \left(2 k_{c n}\right) \approx 1 \\
\sin \left(2 k_{c n}\right) \approx 2 k_{c n}
\end{gathered}
$$

were exploited to obtain (9).

The first term of the right-hand side (RHS) of (9) is identical to the monopulse ratio which would result from a single point target [12]. The second term of the RHS of (9) causes an angular error by having a nonzero value when the radar is pointed towards the jammer because all $k_{c n} \propto \cos \left(\theta_{r}\right)$. Retrodirective beacons eliminate the angular error by having a zero cross-eye gain $\left(G_{C N}=0\right)$ to provide a strong return at the centre of the jammer [10], [26]. Cross-eye jammers seek to maximise the angular error by having a large angular separation between the jammer antennas from the radar's perspective $\left(\theta_{e n}\right)$ because $k_{c n} \propto \theta_{e n}$, and ensuring that the cross-eye gain $\left(G_{C N}\right)$ is as large as possible.

The total cross-eye gain is given by [22]

$$
G_{C N}=\mathscr{R} e\left\{\frac{\sum_{n=1}^{N}\left(1-a_{n} e^{j \phi_{n}}\right) A_{n} e^{j \Phi_{n}} s_{r n}}{\sum_{n=1}^{N}\left(1+a_{n} e^{j \phi_{n}}\right) A_{n} e^{j \Phi_{n}}}\right\}
$$

where $N$ is the number of jammer loops, and the jammer baseline ratio is given by

$$
s_{r n}=\frac{\sin \left(2 k_{c n}\right)}{\sin \left(2 k_{c N}\right)} .
$$

The forms of (8), (9) and (12) have slight notational differences to the comparable results in [22] due to the use of the baseline ratio $s_{r n}$ instead of the parameter $d_{c r n}=d_{c n} / d_{c N}$. These changes serve only to simplify the notation and do not otherwise affect the results. Further motivation for the changes can be seen by using (2), (7), and (11) to rewrite (13) as

$$
s_{r n} \approx \frac{k_{c n}}{k_{c N}}=\frac{\theta_{e n}}{\theta_{e N}}=\frac{d_{c n}}{d_{c N}} \frac{\cos \left(\theta_{c n}\right)}{\cos \left(\theta_{c N}\right)} .
$$

The jammer baseline ratio is thus based on the values of $k_{c n}$, which directly affect the error induced in the threat radar. By comparison, $d_{c r n}$ ignores the jammerloop rotations $\left(\theta_{c n}\right)$ and only considers the jammer-loop baselines $\left(d_{c n}\right)$, thereby removing the direct link to the induced angular error.

When there is only one jammer loop $(N=1)$, (12) reduces to the well-known cross-eye gain for single-loop cross-eye jamming [4]-[10]

$$
\begin{aligned}
G_{C} & =\mathscr{R} e\left\{\frac{1-a e^{j \phi}}{1+a e^{j \phi}}\right\} \\
& =\frac{1-a^{2}}{1+a^{2}+2 a \cos (\phi)} .
\end{aligned}
$$

The total cross-eye gain in (12) can be directly compared to the conventional single-loop cross-eye gain in 16 as long as jammer loop $N$ has the largest antenna spacing 
from the perspective of the threat radar $\left(\theta_{e N} \geq \theta_{e n} \forall n\right.$ so $s_{r N}=1$ and $s_{r n} \leq 1 \forall n$ ). While this constraint may not be strictly necessary, it does ensure that the overall baselines are the same for the two cases to allow fair comparisons to be made.

It can be shown that the single-loop cross-eye gain $\left(G_{C}\right)$ always increases as the jammer-channel phase difference $(\phi)$ approaches $180^{\circ}$ because this simultaneously reduces the denominators and increases the numerators of $(15)$ and $(16)$, leading to a higher cross-eye gain [16]. Similar reasoning shows that improving the amplitude matching between the two paths through a jammer loop $(a \rightarrow 1)$ also increases the cross-eye gain. In the limit as the jammer channels approach the ideal match, the cross-eye gain tends to an infinite value $\left(G_{C} \rightarrow \infty\right.$ as $a \rightarrow 1$ and $\phi \rightarrow 180^{\circ}$ ) [4]-[10], [16].

Comparing (12) and (15) shows that increasing the number of jammer loops from 1 to $N$ increases the degrees of freedom available to control the cross-eye gain $\left(G_{C}\right)$ from 2 to $5 N-3\left(a_{n}, \phi_{n}, A_{n}, \Phi_{n}\right.$ and $s_{r n}$ per loop with $s_{r N}=1$ and one value of each of $A_{n}$ and $\Phi_{n}$ being a reference for the others as in $(20)$ ). These additional degrees of freedom allow a reduction of the sensitivity to parameter variations and a reduction of the JSR required from each jammer loop [17]-[22].

The cross-eye gain depends on the phases of each jammer loop $\left(\Phi_{n}\right)$ as shown in (12). These phases are determined by the range from the radar to each of the jammer antennas given by [23]

$$
r_{j n}\left(\theta_{c n}\right)=\sqrt{\left[r+\frac{d_{c n}}{2} \sin \left(\theta_{c n}\right)\right]^{2}+\left[\frac{d_{c n}}{2} \cos \left(\theta_{c n}\right)\right]^{2}}
$$

with positive and negative values of $\theta_{c n}$ corresponding to the further and nearer jammer antennas respectively. The total path length for each jammer loop is

$$
r_{t n}\left(\theta_{c n}\right)=r_{j n}\left(\theta_{c n}\right)+r_{j n}\left(-\theta_{c n}\right),
$$

so the loop phase is given by

$$
\Phi_{n}=\beta r_{t n}
$$

for jammer loop $n$. The different values of $d_{c n}$ and $\theta_{c n}$ for each jammer loop mean that the loop phases will differ, and (12) shows that these phase differences will affect the cross-eye gain $\left(G_{C N}\right)$.

\section{B. Cross-Eye Gain as a Function of Path-Length Phase}

The effect of path-length differences on the performance of a multi-loop retrodirective cross-eye jammer will now be considered. The case with two jammer loops will be considered because additional jammer loops dramatically complicate the analysis without providing significant additional insight. For example, while (12) shows that beacon operation is possible as the values of the path-length phases $\left(\Phi_{n}\right)$ change, the large number of parameters which need to be considered tend to obscure the underlying principles.

The two-loop cross-eye gain is given by

$$
G_{C 2}=\mathscr{R} e\left\{\frac{\left(1-a_{2} e^{j \phi_{2}}\right)+\left(1-a_{1} e^{j \phi_{1}}\right) A e^{j \Phi} s_{r 1}}{\left(1+a_{2} e^{j \phi_{2}}\right)+\left(1+a_{1} e^{j \phi_{1}}\right) A e^{j \Phi}}\right\}
$$

where $A=A_{1} / A_{2}$ and $\Phi=\Phi_{1}-\Phi_{2}$ were introduced without loss of generality. As outlined above, jammer loop 2 (the outer loop) has a baseline ratio of one, while jammer loop 1 (the inner loop) has a baseline ratio of less than one.

The effect of the phase difference between the two jammer loops $(\Phi)$ on the cross-eye gain $\left(G_{C 2}\right)$ can be highlighted by rewriting (20) as

$$
G_{C 2}=\frac{c_{1}+c_{2} \cos (\Phi)+c_{3} \sin (\Phi)}{c_{4}+c_{5} \cos (\Phi)+c_{6} \sin (\Phi)}
$$

with the values of the $c_{n}$ being given by

$$
\begin{gathered}
c_{1}=\left(1-a_{1}^{2}\right) A^{2} s_{r 1}+\left(1-a_{2}^{2}\right) \\
c_{2}=\left(1+a_{1} \cos \left(\phi_{1}\right)-a_{2} \cos \left(\phi_{2}\right)-\right. \\
a_{1} a_{2} \cos \left(\phi_{1}-\phi_{2}\right)+ \\
{\left[1-a_{1} \cos \left(\phi_{1}\right)+a_{2} \cos \left(\phi_{2}\right)-\right.} \\
\left.\left.a_{1} a_{2} \cos \left(\phi_{1}-\phi_{2}\right)\right] s_{r 1}\right) A \\
c_{3}=-\left(a_{1} \sin \left(\phi_{1}\right)+a_{2} \sin \left(\phi_{2}\right)-\right. \\
a_{1} a_{2} \sin \left(\phi_{1}-\phi_{2}\right)- \\
{\left[a_{1} \sin \left(\phi_{1}\right)+a_{2} \sin \left(\phi_{2}\right)+\right.} \\
\left.\left.a_{1} a_{2} \sin \left(\phi_{1}-\phi_{2}\right)\right] s_{r 1}\right) A \\
c_{4}=\left[1+a_{1}^{2}+2 a_{1} \cos \left(\phi_{1}\right)\right] A^{2}+ \\
1+a_{2}^{2}+2 a_{2} \cos \left(\phi_{2}\right) \\
c_{5}=2\left[1+a_{1} \cos \left(\phi_{1}\right)+a_{2} \cos \left(\phi_{2}\right)+\right. \\
\left.a_{1} a_{2} \cos \left(\phi_{1}-\phi_{2}\right)\right] A \\
c_{6}=-2\left[a_{1} \sin \left(\phi_{1}\right)-a_{2} \sin \left(\phi_{2}\right)+\right. \\
\left.a_{1} a_{2} \sin \left(\phi_{1}-\phi_{2}\right)\right] A .
\end{gathered}
$$

The form of 21) is anticipated in light of the fact that the complex exponential $e^{-j \Phi}$ appears in both the numerator and denominator of $G_{C 2}$ in 20 .

Using identical jammer loops $\left(a_{1}=a_{2}=a\right.$ and $\phi_{1}=$ 
$\left.\phi_{2}=\phi\right)$ simplifies the above results to

$$
\begin{gathered}
c_{1}=\left(1-a^{2}\right)\left(1+A^{2} s_{r 1}\right) \\
c_{2}=\left(1-a^{2}\right) A\left(1+s_{r 1}\right) \\
c_{3}=-2 a \sin (\phi) A\left(1-s_{r 1}\right) \\
c_{4}=\left[1+a^{2}+2 a \cos (\phi)\right]\left(1+A^{2}\right) \\
c_{5}=2\left[1+a^{2}+2 a \cos (\phi)\right] A \\
c_{6}=0 .
\end{gathered}
$$

While assuming identical parameters for the two jammer loops does make the results less general, the considerable simplifications achieved by doing so make this assumption justifiable. Comparing (22) to 27) and 28) to 33 shows that the greatest differences are in the parameters $c_{3}$ and $c_{6}$. These parameters are the coefficients of $\sin (\Phi)$ in (21), so the effect of assuming identical jammer loops will be small for similar path lengths $(\Phi \approx 0)$, but will increase as the path-length difference increases.

It is worth noting that the value of the phase difference between the jammer loops $(\Phi)$ depends on a number of factors other than path length. So while $\Phi$ is used to investigate the effects of path-length differences in this work, the results apply to any case where $\Phi$ is varied.

\section{Potential FOR BEACON OPERATION}

Path-length differences can cause a cross-eye jammer to act as a beacon when the cross-eye gain becomes zero $\left(G_{C N}=0\right)$ [23]. This outcome is undesirable as the goal of any jammer is to compromise the operation of a threat rather than to assist it.

The two-loop cross-eye gain is zero when

$$
\begin{aligned}
0= & G_{C 2} \\
= & c_{1}+c_{2} \cos (\Phi)+c_{3} \sin (\Phi) \\
= & c_{1}+\sqrt{c_{2}^{2}+c_{3}^{2}} \times \\
& \quad\left[\cos \left(\theta_{o}\right) \cos (\Phi)+\sin \left(\theta_{o}\right) \sin (\Phi)\right] \\
= & c_{1}+\sqrt{c_{2}^{2}+c_{3}^{2}} \cos \left(\Phi-\theta_{o}\right) \\
\Phi= & \pm \arccos \left(-\frac{c_{1}}{\sqrt{c_{2}^{2}+c_{3}^{2}}}\right)+\theta_{o} \\
= & \pm \theta_{b}+\theta_{o}
\end{aligned}
$$

where

$$
\begin{aligned}
& \cos \left(\theta_{o}\right)=\frac{c_{2}}{\sqrt{c_{2}^{2}+c_{3}^{2}}} \\
& \sin \left(\theta_{o}\right)=\frac{c_{3}}{\sqrt{c_{2}^{2}+c_{3}^{2}}} .
\end{aligned}
$$

The base angle $\left(\theta_{b}\right)$ determines the range of path-length phase differences $(\Phi)$ which avoid beacon operation, while the offset angle $\left(\theta_{o}\right)$ determines where this range of path-length differences is situated.

\section{A. Base Angle}

The base angle $\left(\theta_{b}\right)$ determines the magnitude of the range of path-length phase differences $(\Phi)$ over which beacon operation is impossible, so a value of zero would mean that beacon operation is impossible.

From (38) and (39) the base angle is given by

$$
\theta_{b}=\arccos \left(-\frac{c_{1}}{\sqrt{c_{2}^{2}+c_{3}^{2}}}\right),
$$

and nature of the arccosine function in (38) means beacon operation is avoided when

$$
\begin{aligned}
\left|\frac{c_{1}}{\sqrt{c_{2}^{2}+c_{3}^{2}}}\right| & >1 \\
\left(\frac{c_{2}}{c_{1}}\right)^{2}+\left(\frac{c_{3}}{c_{1}}\right)^{2} & <1
\end{aligned}
$$

where $\left|c_{1}\right|=c_{1}$ because $0 \leq a \leq 1$ and $A>0$ by definition. Substituting (28) to (30) into (44) results in

$$
\left[\frac{A\left(1+s_{r 1}\right)}{1+A^{2} s_{r 1}}\right]^{2}+\left[\frac{2 a \sin (\phi)}{1-a^{2}} \cdot \frac{A\left(1-s_{r 1}\right)}{1+A^{2} s_{r 1}}\right]^{2}<1
$$

which expresses the result in terms of the jammer parameters $a, \phi, A$ and $s_{r 1}$.

Setting $A=0$ or $A \rightarrow \infty$ makes the left-hand side (LHS) of (45) zero, thereby satisfying the inequality. These two conditions correspond to disabling one of the jammer loops, so this result is anticipated as a single jammer loop is not affected by path-length differences. While obvious, this outcome provides a measure of validation of the results.

Having equal jammer-loop magnitudes $(A=1)$ is reasonable because similar hardware is likely to be used for all jammer loops. However, this makes the first term of the LHS of (45) equal to one, thereby ensuring that the inequality is always violated. Path-length differences can thus always cause a multi-loop retrodirective crosseye jammer to act as a beacon when the jammer loops have identical magnitudes.

The first term of the LHS of (45) is also equal to one when $A=1 / s_{r 1}$. The numerator of (20) shows that this magnitude selection means that all jammer loops have the same contribution to the difference-channel signal received by the threat radar. So adjusting the loop gain to compensate for loop baseline differences also means that path-length differences will always be capable of turning a multi-loop retrodirective cross-eye jammer into a beacon. 
The largest value of the first term of the LHS of 45 is obtained when

$$
\begin{aligned}
0 & =\frac{d}{d A} \frac{A\left(1+s_{r 1}\right)}{1+A^{2} s_{r 1}} \\
A & =\frac{1}{\sqrt{s_{r 1}}}
\end{aligned}
$$

where the possible negative solution was ignored because $A>0$ by definition. The largest value of the first term of the LHS of 45] is thus given by

$$
\left.\frac{A\left(1+s_{r 1}\right)}{1+A^{2} s_{r 1}}\right|_{A=\frac{1}{\sqrt{s_{r 1}}}}=\frac{1+s_{r 1}}{2 \sqrt{s_{r 1}}}
$$

which is greater than one unless the baseline ratios are equal $\left(s_{r 1}=1\right)$ when its value is one. The jammer baseline differences can thus not be used to guarantee that beacon operation is impossible.

Taken together, the above observations mean that $1<A<1 / s_{r 1}\left(s_{r 1} \leq 1\right.$ by definition) makes the first term of the LHS of (45) greater than one, thereby ensuring that the inequality cannot be satisfied. Magnitude matches outside this range are thus required to avoid beacon operation if the second term of the LHS of 45) is zero. Unfortunately, this range of values is potentially important because the sum-channel returns from the two jammer loops are equal when $A=1$, while the difference-channel returns are equal when $A=1 / s_{r 1}$. Values outside this range will thus lead to one jammer loop having an increasingly dominant effect on the jammer, eventually defeating the point of having multiple jammer loops.

The second term of the LHS of (45) consists of two factors, one of which depends on the jammer-channel parameters $(a$ and $\phi)$, while the other depends on the match between the jammer loops ( $A$ and $\left.s_{r 1}\right)$.

The factor $2 a \sin (\phi) /\left(1-a^{2}\right)$ will be small because $\phi \approx 180^{\circ}$ in cross-eye jamming. However, the amplitude match required for cross-eye jamming $(a \approx 1)$ makes $a /\left(1-a^{2}\right)$ extremely large, potentially counteracting the effect of the small $\sin (\phi)$. Reducing this factor thus requires a substantial mismatch between the two directions through the jammer $(a \ll 1)$ to satisfy the inequality in (45). While such mismatches are desirable to reduce the effect of tolerances [10], [16] and platform skin return [14], [15], [25], excessive mismatches will render a cross-eye jammer ineffective by reducing the cross-eye gain $\left(G_{C N}\right)$.

The value of the jammer-channel phase difference $(\phi)$ which serves as the boundary beyond which beacon

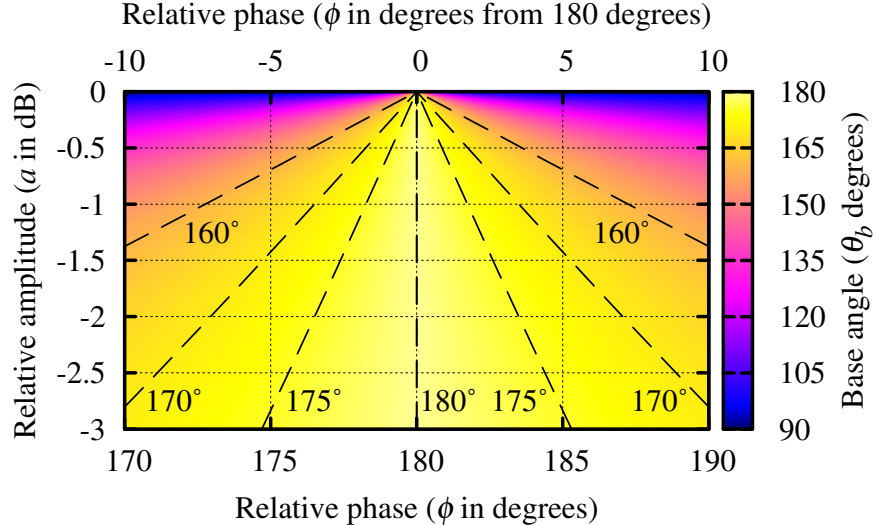

(a)

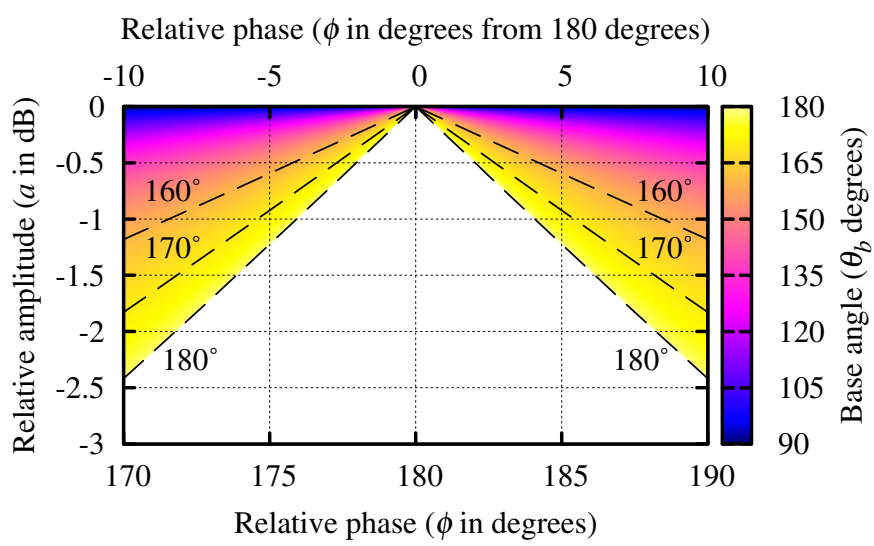

(b)

Fig. 3. The base angle as a function of the jammer-channel parameters $(a$ and $\phi)$ when (a) $A=0 \mathrm{~dB}$ and $s_{r 1}=0.5$, and (b) $A=-0.5 \mathrm{~dB}$ and $s_{r 1}=0.5$.

operation is avoided is obtained by rewriting (45) as

$$
|\sin (\phi)|<\frac{\left(1-a^{2}\right) \sqrt{\left(1-A^{2}\right)\left(1-A^{2} s_{r 1}^{2}\right)}}{2 a A\left(1-s_{r 1}\right)}
$$

which is always positive because $0 \leq a \leq 1$ and $s_{r 1}<1$ by definition. In agreement with above observations, the RHS of 49 is imaginary when $1<A<1 / s_{r 1}$, showing that beacon operation cannot be avoided under these conditions. While similar equations for the remaining parameters would be valuable, the results are too complex to be useful, and as outlined above, no value of $s_{r 1}$ can guarantee that beacon operation is impossible.

Fig. 3 shows the relationship between the base angle $\left(\theta_{b}\right)$ and the jammer-channel parameters $(a$ and $\phi)$ for two values of the match between the jammer loops ( $A$ and $\left.s_{r 1}\right)$. The white region in Fig. 3 indicates where the base angle does not exist making beacon operation impossible.

The most significant observation from Fig. 3 is that the minimum value of the base angle is $90^{\circ}$ in all cases. There is thus a wide range of path-length phase 


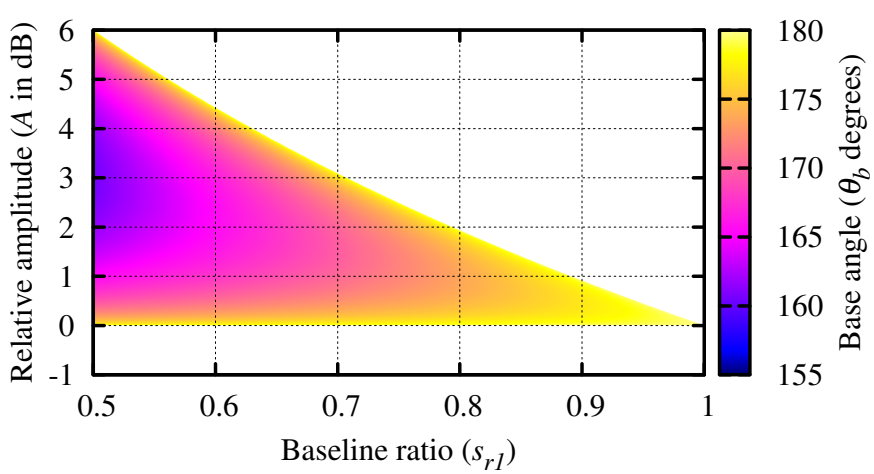

(a)

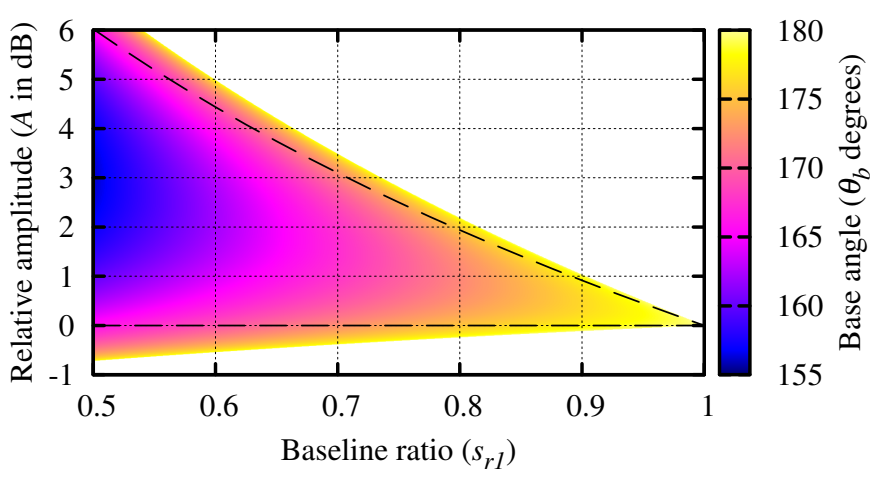

(b)

Fig. 4. The base angle as a function of the jammer-loop parameters ( $A$ and $s_{r 1}$ ) when (a) $a=-1 \mathrm{~dB}$ and $\phi=180^{\circ}$, and (b) $a=-1 \mathrm{~dB}$ and $\phi=175^{\circ}$. The dashed lines in (b) show the boundaries of the coloured region in (a)

differences $(\Phi)$ over which the jammer will not act as a beacon.

Fig. 3 also shows that that the base angle $\left(\theta_{b}\right)$ increases rapidly as jammer-channel amplitudes become increasingly mismatched $(a \rightarrow 0)$. This means that even small channel-amplitude mismatches can significantly decrease the likelihood of beacon operation.

Comparing Figs 3(a) and 3(b) shows that even a small amplitude mismatch between the jammer loops ( $A=-0.5 \mathrm{~dB}$ in this case) can lead to large increases in the range of jammer-channel parameters $(a$ and $\phi)$ over which beacon operation is impossible.

While not shown in Fig. 3, increasing the jammer baseline ratio $\left(s_{r 1}\right)$ further reduces the likelihood of beacon operation. Usefully, (12) shows that a larger baseline ratio also leads to increased cross-eye gain $\left(G_{C N}\right)$ [22]. The small baseline ratio used in Fig. 3 is thus likely to be conservative, with larger base angles $\left(\theta_{b}\right)$ being encountered in practice.

Fig. 4 investigates the effect of the jammer-loop amplitude match $(A)$ and the jammer baseline ratio $\left(s_{r 1}\right)$ on the base angle.

Fig. 4(a) only considers the first term of the LHS of (45) as the second term is zero because $\phi=180^{\circ}$. As predicted, values of $A$ which are less than 1 or greater than $1 / s_{r 1}$ avoid beacon operation. Fig. 4 confirms that large amplitude mismatches are required to avoid beacon operation when the inner loop has the greater amplitude $(A>1)$, though the required mismatch decreases as the baseline ratio increases $\left(A \rightarrow 1\right.$ as $\left.s_{r 1} \rightarrow 1\right)$. By comparison, only a small mismatch is required when the outer loop has the greater amplitude $(A<1)$.

The effect of adding the second term of the LHS of (45) is shown in Fig. 4(b), It can be seen that greater mismatches are required than in Fig. 4(a) because the second term contributes to causing beacon operation. The additional mismatch increases as the baseline ratio decreases $\left(s_{r 1} \rightarrow 0\right)$.

The additional mismatch required in Fig. 4(b) is surprisingly small because the jammer-channel amplitude mismatch is large $(a \ll 1)$ while the jammerchannel phase mismatch is small $\left(\phi \approx 180^{\circ}\right)$. A greater jammer amplitude mismatch $(a \rightarrow 0)$ would decrease the additional amplitude mismatch required between the jammer loops and cause the results to tend towards those in Fig. 4(a), while the opposite would happen for a better amplitude match $(a \rightarrow 1)$. Conversely, a better jammerchannel phase match $\left(\phi \rightarrow 180^{\circ}\right)$ is required to decrease the additional mismatch required.

Again, the base angle obtained when beacon operation is possible is extremely large with the axes of both graphs in Fig. 4 starting at $155^{\circ}$. As before, this observation indicates that a broad range of path-length phase differences can be tolerated without the jammer acting as a beacon.

By way of validation, it is noted that the results in [23] support the observation that large path-length differences are required to cause beacon operation.

\section{B. Offset Angle}

The centre of the range of path-length phase differences $(\Phi)$ over which beacon operation is impossible is determined by the offset angle $\left(\theta_{o}\right)$.

From (40) and (41), the offset angle is given by

$$
\begin{aligned}
\theta_{o} & =\arctan \left(\frac{c_{3}}{c_{2}}\right) \\
& =\arctan \left(\frac{2 a \sin (\phi)}{1-a^{2}} \cdot \frac{1-s_{r 1}}{1+s_{r 1}}\right) .
\end{aligned}
$$

An important observation from (51) is that the offset angle is not affected by the jammer-loop amplitude match $(A)$. As highlighted in Section III-A $2 a \sin (\phi) /\left(1-a^{2}\right)$ will be small as long as the jammerchannel phases are well matched $\left(\phi \approx 180^{\circ}\right)$, and there is a mismatch between the amplitudes of the two channels 


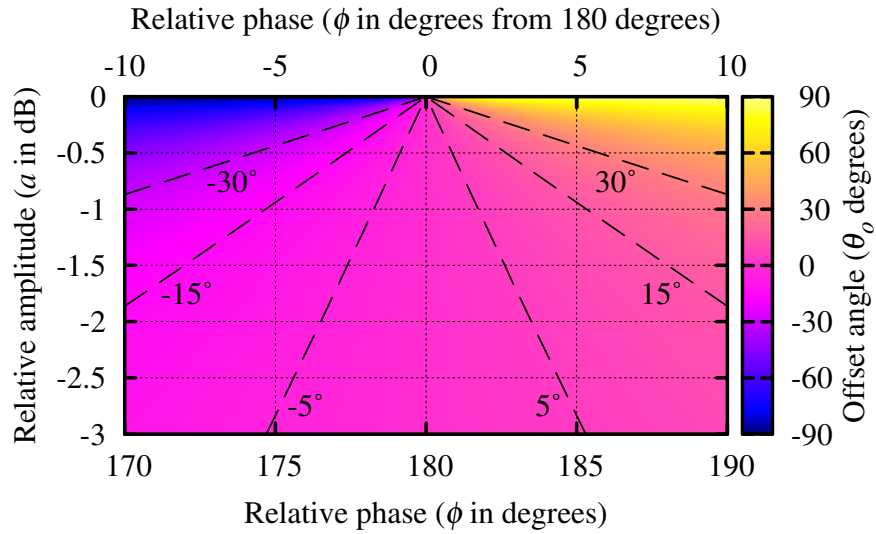

Fig. 5. The offset angle as a function of the jammer-channel parameters $(a$ and $\phi)$ for $s_{r 1}=0.5$.

$(a \ll 1)$. Furthermore, $0 \leq s_{r 1} \leq 1$, so the second factor above will always be less than 1 . It is thus reasonable to anticipate that the offset angle $\left(\theta_{o}\right)$ will be small for practical cross-eye jammer systems.

Equation 51 can be rewritten as

$$
\sin (\phi)=\frac{1-a^{2}}{2 a} \cdot \frac{1+s_{r 1}}{1-s_{r 1}} \tan \left(\theta_{o}\right)
$$

and

$$
s_{r 1}=\frac{2 a \sin (\phi)+\left(1-a^{2}\right) \tan \left(\theta_{o}\right)}{2 a \sin (\phi)-\left(1-a^{2}\right) \tan \left(\theta_{o}\right)}
$$

which allow the values of jammer-channel phase $(\phi)$ and baseline ratio $\left(s_{r 1}\right)$ to achieve a specified offset angle $\theta_{o}$ to be determined.

Fig. 5 shows the effect of jammer-channel parameters ( $a$ and $\phi$ ) on the offset angle. The most important observation from Fig. 5 is that the offset angle is small for the realistic range of jammer-channel parameters shown. The offset angles shown in Fig. 5 are larger than would be encountered in practice as the baseline ratio used is small $\left(s_{r 1}=0.5\right)$ and the offset angle decreases as the jammer baselines converge $\left(s_{r 1} \rightarrow 1\right)$.

There is thus a large range of path-length phase differences $(\Phi)$ around zero where a multi-loop retrodirective cross-eye jammer will not act as a beacon.

\section{Cross-Eye Gain Sensitivity}

The sensitivity of the cross-eye gain $\left(G_{C N}\right)$ to the path-length phase $(\Phi)$ can be quantified by the gradient of the total cross-eye gain to path-length phase. Small gradients are desirable as they indicate that the cross-eye gain will not vary significantly as the path-length phase varies $\left(G_{C N}\right.$ has a low sensitivity to $\left.\Phi\right)$. The angular error induced in the threat radar is determined by the cross-eye gain (see Section II-A), so a stable cross-eye gain implies a stable angular error.

The gradient of the two-loop cross-eye gain $\left(G_{C 2}\right)$ with respect to the loop phase difference $(\Phi)$ is given by

$$
\begin{aligned}
\frac{d G_{C 2}}{d \Phi}= & \frac{-c_{2} \sin (\Phi)+c_{3} \cos (\Phi)}{c_{4}+c_{5} \cos (\Phi)+c_{6} \sin (\Phi)}+ \\
& \frac{c_{5} \sin (\Phi)-c_{6} \cos (\Phi)}{\left[c_{4}+c_{5} \cos (\Phi)+c_{6} \sin (\Phi)\right]^{2}} \times \\
=- & {\left[c_{1}+c_{2} \cos (\Phi)+c_{3} \sin (\Phi)\right] } \\
& \frac{2 a \sin (\phi)\left[2 A+\left(1+s_{r 1}\right) \times\right.}{\left[1+a^{2}+2 a \cos (\phi)\right] \times \cdots} \\
& \frac{\cdots\left(1-a^{2}\right)\left(1-A^{2}\right) \sin (\Phi)}{\cdots\left[1+A^{2}+2 A \cos (\Phi)\right]^{2}} .
\end{aligned}
$$

The first line of (55) shows that the magnitude of the cross-eye gain gradient decreases as the jammer baselines converge $\left(s_{r 1} \rightarrow 1\right)$. This result is anticipated as equal baselines inherently mean that there is no pathlength difference. Equation (14) demonstrates that the baseline ratio $\left(s_{r 1}\right)$ depends on both the jammer baselines $\left(d_{c n}\right)$ and the jammer rotations $\left(\theta_{c n}\right)$, so it is possible for jammer loops with different baselines to have equal baseline ratios $\left(s_{r n}=1\right)$ for certain jammer rotations.

The numerator of the second line of 55) can be expected to be small when the jammer-channel phases are well matched $\left(\phi \approx 180^{\circ}\right)$. Conversely, improving the jammer-channel amplitude matches $(a \rightarrow 1)$ will increase the magnitude of this term. The numerator of the last line of 55 will be small when the jammer channel amplitudes are well matched $(a \approx 1)$.

The first term in the denominator of 55 ) is the squared magnitude of the sum-channel return for each of the jammer channels. A cross-eye jammer achieves a large angular error in part by reducing the magnitude of the sum-channel return, so this factor will be small for wellmatched jammer channels $\left(a \approx 1\right.$ and $\left.\phi \approx 180^{\circ}\right)$. This factor will thus lead to large gradients unless the jammerchannel amplitudes are mismatched $(a \ll 1)$.

The last term in the denominator of (55) assumes its largest value of $(1+A)^{4}$ when there is no pathlength difference $(\Phi=0)$ and its smallest value of $(1-A)^{4}$ when the path-length phase difference is $180^{\circ}$ $(\Phi=\pi)$. The large value of this term for small pathlength differences means that the cross-eye gain $\left(G_{C 2}\right)$ will change slowly in response to path-length differences. Similar reasoning suggests that the cross-eye gain will be sensitive to path-length difference changes when the path-length difference is near $180^{\circ}$. Furthermore, decreasing the amplitude of the inner loop $(A \rightarrow 0)$ will reduce the magnitude of the variation of this factor, 


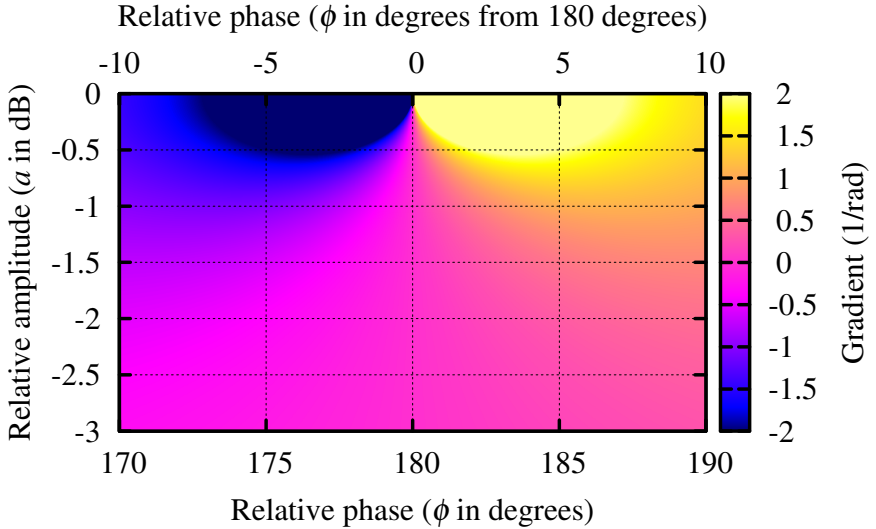

(a)

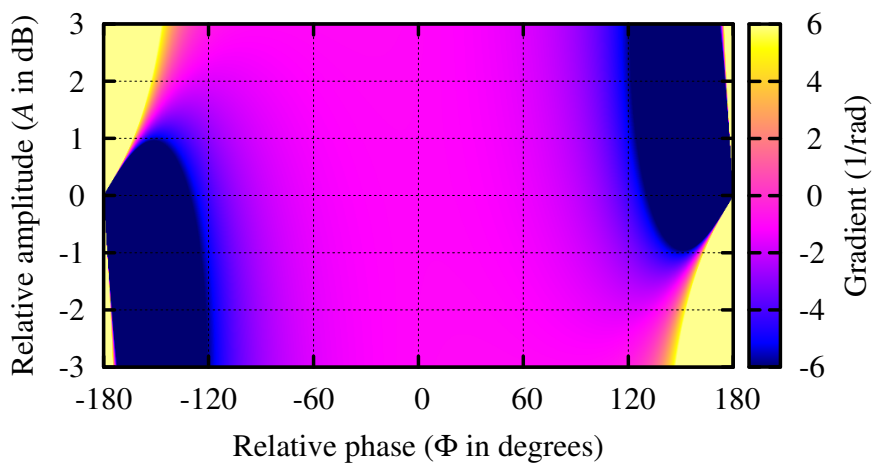

(b)

Fig. 6. The gradient of the cross-eye gain $\left(G_{C 2}\right)$ when (a) $A=0 \mathrm{~dB}$ $\Phi=0$ and $s_{r 1}=0.5$, and (b) $a=-1 \mathrm{~dB}, \phi=175^{\circ}$ and $s_{r 1}=0.5$.

thereby leading to more consistent cross-eye gain values across the full range of path-length differences.

The first and second lines of (55) and the variation in the denominator of the last line of (55) all decrease as the amplitude of the inner loop decreases $(A \rightarrow 0)$, while the numerator of the last line of (55) will be zero when the loop amplitudes are matched $(A=1)$. The smallest cross-eye gain gradient is thus expected when the amplitude of the inner loop is slightly smaller than that of the outer loop $(A<1$ and $A \approx 1)$. Significantly, Section $\amalg$ shows that this condition will also ensure that beacon operation is impossible.

Fig. 6 considers the cross-eye gain gradient as a function of jammer-channel parameters ( $a$ and $\phi)$ in Fig. 6(a) and the jammer-loop parameters $(A$ and $\Phi)$ in Fig. 6(b),

The primary conclusion of Fig. 6(a) is that the rate of change of the cross-eye gain is small unless the jammerchannel amplitude match is good $(a \approx 1)$. Additionally, the gradient is lower when the jammer-channel phase matching is $\operatorname{good}\left(\phi \approx 180^{\circ}\right)$.

Fig. 6(b) shows that the sensitivity to both the jammerloop parameters $(A$ and $\Phi)$ is extremely low when the path-length difference is small $(\Phi \approx 0)$. Perhaps more

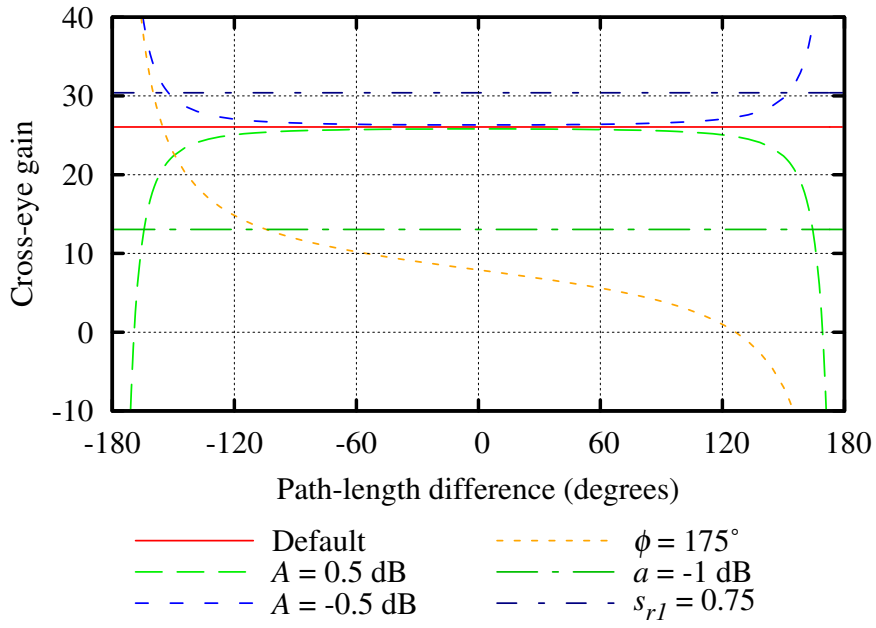

(a)

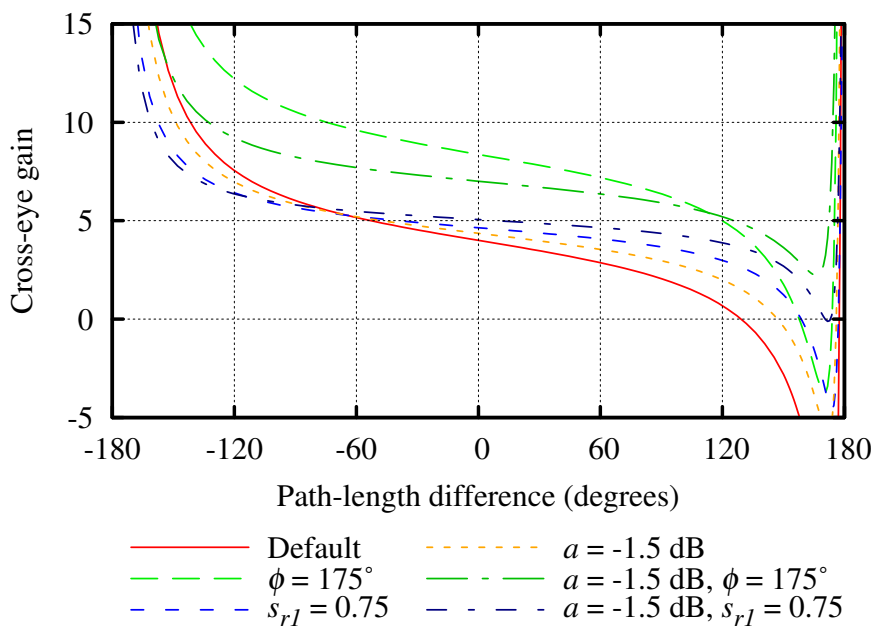

(b)

Fig. 7. The total cross-eye gain for a number of two-loop jammer parameters. The default parameters for each figure are (a) $a=-0.5 \mathrm{~dB}$ $\phi=180^{\circ}, A=0 \mathrm{~dB}$ and $s_{r 1}=0.5$, and (b) $a=-1 \mathrm{~dB}, \phi=170^{\circ}$, $A=-0.5 \mathrm{~dB}$ and $s_{r 1}=0.5$.

importantly, the gradient is extremely small over the vast majority of the range of parameters shown. However, the gradient does increase rapidly as the path-length phase difference approaches $\pm 180^{\circ}$. Again, these conclusions are supported by the results in [23].

\section{Total CRoss-Eye GAin}

The total cross-eye gain for a number of two-loop jammers is evaluated below to demonstrate the value of the preceding analyses.

Fig. 7 shows the total cross-eye gain $\left(G_{C 2}\right)$ for a number of different jammer parameters. In each graph, the specified default parameters are modified to investigate specific aspects of the total cross-eye gain.

The default parameters in Fig. 7(a) represent well matched jammer channels $\left(a \approx 1\right.$ and $\left.\phi=180^{\circ}\right)$ and 
jammer-loop amplitudes $(A=1)$. The cross-eye gain $\left(G_{C 2}\right)$ is thus expected to be large, though the small baseline of the inner loop $\left(s_{r 1}=0.5\right)$, will reduce the gain somewhat $]^{1}$

The overriding conclusion from Fig. 7 is that pathlength phase differences $(\Phi)$ have a significant effect on the operation of a multi-loop cross-eye jammer. While it is possible to remove variations due to path-length differences for specific parameter values, the required parameters are not always conducive to achieving high cross-eye gain $\left(G_{C N}\right)$, and even small deviations from the required values will again introduce sensitivity to path-length differences.

Many of the curves in Fig. 7 are not symmetrical, while all the curves in the comparable figure in [23] are symmetrical. This difference arises as each of the curves in Fig. 7 is for a specific combination of jammer parameters, while the curves in [23] are statistical distributions. For each curve in Fig. 7 there is a identical curve which is flipped around $\Phi=0$ when the jammer-channel phase $\phi=\pi-\phi_{o}$ is replaced by $\phi=\pi+\phi_{o}$.

The gradient of the cross-eye gain in Fig. 7 is small when the path-length phase difference is small $(\Phi \approx 0)$, but increases as the phase difference approaches $180^{\circ}$ $\left(\Phi \rightarrow \pm 180^{\circ}\right)$. This behaviour was predicted in Section IV.

The default parameters in Fig. 7(a) will result in a zero cross-eye gradient for all values of path-length phase difference $(\Phi)$ because the jammer-channel phase and jammer-loop amplitudes are well matched $\left(\phi=180^{\circ}\right.$ and $A=1)$. This parameter combination ensures that (55) is always zero.

Varying the jammer-loop amplitude $(A)$ in Fig. 7(a) means that the gradient is no longer zero at all angles. Increasing the amplitude of the inner loop $(A>1)$ will lead to the possibility of beacon operation $\left(G_{C N}=0\right)$ for certain values of the path-length phase difference $(\Phi)$, while decreasing the inner-loop amplitude $(A<1)$ prevents beacon operation $\left(G_{C N} \neq 0\right)$. Even small amplitude variations $(-0.5 \mathrm{~dB} \leq A \leq 0.5 \mathrm{~dB})$ can thus lead to rather different results for large path-length phase differences $\left(\Phi \approx \pm 180^{\circ}\right)$. This result agrees with the analysis in Section III-A

Adjusting the jammer-channel phase away from the ideal condition $\left(\phi \neq 180^{\circ}\right)$ will both reduce the crosseye gain $\left(G_{C 2}\right)$ and lead to a non-zero gradient as seen from the case where $\phi=175^{\circ}$ in Fig. 7(a), Again, this result is predicted by the analysis in Section IV] This is the only case in Fig. 7(a) where the cross-eye gain is not

\footnotetext{
${ }^{1}$ For example, the total cross-eye gain of the default multi-loop case is 26.1 , while a single-loop cross-eye jammer with the same jammer-channel parameters $(a$ and $\phi$ ) would have a gain of 34.8 .
}

symmetrical around $\Phi=0$ because it is the only case for which the offset angle $\left(\theta_{o}\right)$ is not zero (see Section III-B).

A poorer jammer-channel amplitude match $(a \ll 1)$ is seen to reduce the cross-eye gain $\left(G_{C 2}\right)$ in Fig. $7(\mathrm{a})$ but not to further affect the results. This is because the other parameter values mean that the jammer-channel amplitude (a) does not affect either the possibility of beacon operation or the gradient, as shown in Sections III and IV

The final parameter change in Fig. 7(a) is to increase the inner-loop baseline $\left(d_{c 1}\right)$ to increase the baseline ratio $\left(s_{r 1}\right)$. Sections III and IV again show that the values of the other parameters mean that the baseline ratio does not affect the possibility of beacon operation or the gradient. However, (20) shows that the cross-eye gain $\left(G_{C 2}\right)$ increases as the baseline ratio increases [23], and this is borne out by the relevant curve in Fig. 7(a).

Fig. 7(b) shows a case where the default jammerchannel match $\left(a=-1.0 \mathrm{~dB}\right.$ and $\left.\phi=170^{\circ}\right)$ is significantly poorer than that in Fig. 7(a) The main consequence of the poorer jammer-channel match is a lower cross-eye gain $\left(G_{C 2}\right)$. As expected, improving the jammer-channel phase match $\left(\phi \rightarrow 180^{\circ}\right)$ is shown to increase the gain. Perhaps more importantly, the improved phase match also has the benefit of increasing the base angle or even making beacon operation impossible in Fig. 7(b),

Surprisingly, a worse jammer-channel amplitude match $(a \rightarrow 0)$ actually leads to a higher cross-eye gain $\left(G_{C 2}\right)$ for the majority of path-length phase differences $(\Phi)$ in two of the three cases shown in Fig. 7(b). This result is counterintuitive because better jammer-channel amplitude matches $(a \rightarrow 1)$ usually produce higher crosseye gain. The fact that this is a two-loop cross-eye jammer makes the effect of parameter variations more complex. Importantly, increasing the jammer-channel amplitude mismatch $(a \rightarrow 0)$ increases the base angle $\left(\theta_{b}\right)$ for $\phi=170^{\circ}$ and makes beacon operation impossible when $\phi=175^{\circ}$, thereby supporting the observations in Section $\amalg$ II-A

Increasing the baseline ratio $\left(s_{r 1}\right)$ in Fig. 7(b) increases the cross-eye gain $\left(G_{C 2}\right)$ across the majority of path-length phase mismatches $(\Phi)$. This is a result of a number of factors including the increase in the cross-eye gain, the increase in the base angle $\left(\theta_{b}\right)$, and a decrease in the offset angle $\left(\theta_{o}\right)$ which combine to make beacon operation less likely as outlined in Section III, and to reduce the gradient as described in Section IV

Fig. 8 shows the result of $10^{6}$ evaluations of the crosseye gain $\left(G_{C 2}\right)$ for the parameters uniformly distributed over the ranges listed below

- $a_{1}$ and $a_{2}:-1.5 \mathrm{~dB} \pm 0.5 \mathrm{~dB}$, 


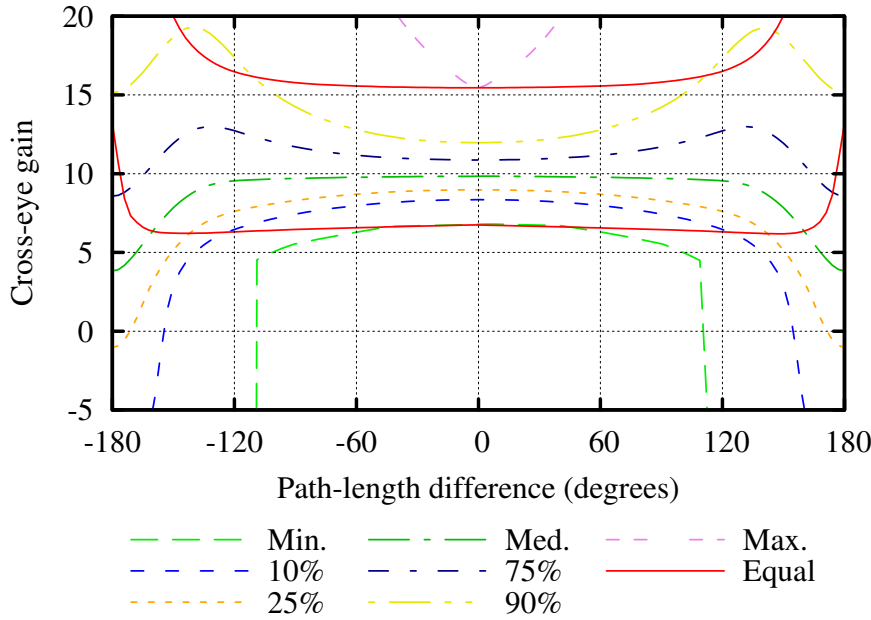

Fig. 8. The results of a Monte-Carlo evaluation of the effect of non-identical jammer parameters for the parameter values provided in the text.

- $\phi_{1}$ and $\phi_{2}: 180^{\circ} \pm 5^{\circ}$,

- $A$ : $-1.5 \mathrm{~dB} \pm 0.5 \mathrm{~dB}$, and

- $\Phi$ : value on graph $\pm 5^{\circ}$, and

- $s_{r 1}: 0.75$.

These parameters were selected as they guarantee that beacon operation is impossible when the jammer-channel parameters are identical $\left(a_{1}=a_{2}\right.$ and $\left.\phi_{1}=\phi_{2}\right)$. A uniform distribution was used as it increases the likelihood of obtaining extreme parameter values.

The curves labelled "Equal" in Fig. 8 show the largest and smallest equal-parameter cross-eye gains $\left(G_{C 2}\right.$ with $a_{1}=a_{2}$ and $\phi_{1}=\phi_{2}$ ). The remaining curves allow different jammer-channel parameters for the loops $\left(a_{1} \neq\right.$ $a_{2}$ and $\phi_{1} \neq \phi_{2}$ ) to reflect how tolerances will affect the parameters in practice. The labels of these curves indicate the proportion of the results which are below that curve. For example, a quarter of the results have a cross-eye gain below the curve labelled " $25 \%$."

Fig. 8 shows that beacon operation is possible when the jammer-channel parameters differ $\left(a_{1} \neq a_{2}\right.$ and $\left.\phi_{1} \neq \phi_{2}\right)$ even though this is not possible for equal jammer-channel parameters $\left(a_{1}=a_{2}\right.$ and $\left.\phi_{1}=\phi_{2}\right)$. However, the equal-parameter results are still useful. While significant variations are seen at large path-length phase differences $\left(\Phi \approx \pm 180^{\circ}\right)$, the results are similar for smaller path-length phase differences $(\Phi \approx 0)$, which are more common in realistic cross-eye jamming scenarios [23]. And while beacon operation is not avoided when the jammer-channel parameters differ, the range of path-length differences over which beacon operation is avoided is still large. Furthermore, while beacon operation is impossible when $|\Phi|<74^{\circ}$ for the parameters used in [23], this range is extended to $|\Phi|<112^{\circ}$ by the above parameters, an improvement of over $50 \%$.

\section{CONCLUSION}

The effect of path-length differences on the operation of multi-loop retrodirective cross-eye jammers is explored. The analysis is complicated by the fact that each additional cross-eye jammer loop increases the number of degrees of freedom of the system, so only two-loop cross-eye jammers with equal jammer-channel parameters $\left(a_{1}=a_{2}\right.$ and $\left.\phi_{1}=\phi_{2}\right)$ are considered in detail.

The most important conclusion is that path-length differences can have a major effect on the operation of a multi-loop cross-eye jammer. While it is possible to identify combinations of parameters which eliminate path-length dependency, even small variations from these required parameter values reintroduce significant sensitivity to path-length differences.

In certain circumstances, path-length differences can even lead to the undesirable situation where a cross-eye jammer acts as a beacon. Fortunately, it is shown that beacon operation can be avoided by a careful selection of the jammer parameters. The likelihood of beacon operation is reduced when

- the baselines are similar (all $d_{c n} \rightarrow d_{c N}$, so $\left.s_{r n} \rightarrow 1\right)$,

- the jammer-channel amplitudes are mismatched $\left(a_{n} \rightarrow 0\right)$,

- the jammer-channel phase difference is close to $180^{\circ}\left(\phi_{n} \rightarrow 180^{\circ}\right)$,

- the jammer effect is dominated by the one of the jammer loops (outer loop of a two-loop jammer when $A<1$ and the inner loop $\left.A>1 / s_{r 1}\right)$, and

- the path-length phase difference is small $\left(\Phi_{n} \rightarrow 0\right)$.

Unfortunately, some of these parameter values reduce the cross-eye gain, thereby reducing the angular error which is induced in the threat radar. There is thus a compromise between achieving high cross-eye gain and avoiding beacon operation.

The gradient of the cross-eye gain with respect to path-length variations was considered as a figure of merit to quantify how sensitive a two-loop cross-eye jammer is to path-length differences. Fortunately, the magnitude of this gradient is low for a broad range of jammer parameters, demonstrating that sensitivity to path-length differences can be reduced through careful system design. Usefully, the parameter values which reduce this gradient are the same as those listed above to minimise the likelihood of beacon operation. The one change is that the gradient magnitude is a highly nonlinear function of path-length phase difference and increases rapidly as the phase difference approaches $\pm 180^{\circ}\left(\Phi \rightarrow \pm 180^{\circ}\right)$.

Finally, Monte-Carlo simulations were used to eval- 
uate the cross-eye gain $\left(G_{C 2}\right)$ of a two-loop cross-eye jammer whose parameter values were selected according to the guidelines listed above. Beacon operation is still possible despite the parameter values being chosen to avoid beacon operation. This is a result of the fact that the analysis was performed under the assumption of equal jammer-channel parameters $\left(a_{1}=a_{2}\right.$ and $\phi_{1}=\phi_{2}$ ), while the simulations considered the practical case where tolerances cause the parameter values to differ $\left(a_{1} \neq a_{2}\right.$ and $\left.\phi_{1} \neq \phi_{2}\right)$. However, use of the guidelines listed above allowed the range of path-length differences $(\Phi)$ for which beacon operation is avoided to be increased by more than $50 \%$ over previouslypublished results. This dramatic improvement clearly demonstrates the value of the analysis as the guidelines used were based on the insight provided by the analysis.

\section{ACKNOWLEDGMENT}

The author wishes to express his sincere thanks to the anonymous reviewers for their insightful comments and valuable suggestions.

\section{REFERENCES}

[1] P. K. Shizume, "Angular deception countermeasure system," U.S.A. Patent 4117484 , September 26, 1978.

[2] B. L. Lewis and D. D. Howard, "Security device," U.S.A. Patent 4006478 , Feb. 1, 1977.

[3] F. Neri, "Experimental testing on cross-eye jamming," in $A O C$ Int. Symp. Conf., Las Vega, USA, 2000.

[4] S. A. Vakin and L. N. Shustov, "Principles of jamming and electronic reconnaissance - Volume I," U.S. Air Force, Tech. Rep. FTD-MT-24-115-69, AD692642, 1969.

[5] L. B. Van Brunt, Applied ECM. EW Engineering, Inc., 1978, vol. 1.

[6] A. Golden, Radar Electronic Warfare. AIAA Inc., 1987.

[7] D. C. Schleher, Electronic warfare in the information age. Artech House, 1999.

[8] F. Neri, Introduction to Electronic Defense Systems, 2nd ed. Rayleigh, USA: SciTech Publishing, 2006.

[9] W. P. du Plessis, J. W. Odendaal, and J. Joubert, "Extended analysis of retrodirective cross-eye jamming," IEEE Trans. Antennas Propag., vol. 57, no. 9, pp. 2803-2806, Sept. 2009.

[10] W. P. du Plessis, "A comprehensive investigation of retrodirective cross-eye jamming," Ph.D. dissertation, University of Pretoria, 2010.

[11] J. W. Wright, "Radar glint - a survey," Electromagnetics, vol. 4, no. 2, pp. 205-227, Jan. 1984.

[12] S. M. Sherman and D. K. Barton, Monopulse principles and techniques, 2nd ed. Artech House, 2011.

[13] F. Neri, "Anti-monopulse jamming techniques," in Proc. 2001 SBMO/IEEE MTT-S Microw. Optoelectronics Conf., vol. 2, 2001, pp. 45-50.
[14] W. P. du Plessis, "Statistical skin-return results for retrodirective cross-eye jamming," IEEE Trans. Aerosp. Electron. Syst., revision submitted on 11 Jan. 2017.

[15] W. P. du Plessis, "Limiting apparent target position in skinreturn influenced cross-eye jamming," IEEE Trans. Aerosp. Electron. Syst., vol. 49, no. 3, pp. 2097-2101, July 2013.

[16] W. P. du Plessis, J. W. Odendaal, and J. Joubert, “Tolerance analysis of cross-eye jamming systems," IEEE Trans. Aerosp. Electron. Syst., vol. 47, no. 1, pp. 740-745, Jan. 2011.

[17] C. Musso and C. Curt, "Robustness of a new angular countermeasure," in Radar 97, 14-16 Oct. 1997, pp. 415-419.

[18] N. M. Harwood, W. N. Dawber, V. A. Kluckers, and G. E. James, "Multiple-element crosseye," IET Radar Sonar Nav., vol. 1, no. 1, pp. 67-73, Feb. 2007.

[19] T. Liu, X. Wei, and L. Li, "Multiple-element retrodirective cross-eye jamming against amplitude-comparison monopulse radar," in Int. Conf. Signal Process. (ICSP), HangZhou, China, Oct 2014, pp. 2135-2140.

[20] S. Liu, C. Dong, J. Xu, G. Zhao, and Y. Zhu, "Analysis of rotating cross-eye jamming," IEEE Antennas Wireless Propag. Lett., vol. 14, pp. 939-942, Dec 2015.

[21] T. Liu, D. Liao, X. Wei, and L. Li, "Performance analysis of multiple-element retrodirective cross-eye jamming based on linear array," IEEE Trans. Aerosp. Electron. Syst., vol. 51, no. 3, pp. 1867-1876, July 2015.

[22] W. P. du Plessis, "Cross-eye gain in multi-loop retrodirective cross-eye jamming," IEEE Trans. Aerosp. Electron. Syst., vol. 52, no. 2, pp. 875-882, April 2016.

[23] W. P. du Plessis, "Path-length effects in multiloop retrodirective cross-eye jamming," IEEE Antennas Wireless Propag. Lett., vol. 15, pp. 626-629, March 2016.

[24] W. P. du Plessis, "Modelling monopulse antenna patterns," in Saudi Int. Electron. Commun. Photon. Conf. (SIECPC), Riyadh, Saudi Arabia, 27-30 April 2013, pp. 1-5.

[25] W. P. du Plessis, "Platform skin return and retrodirective crosseye jamming," IEEE Trans. Aerosp. Electron. Syst., vol. 48, no. 1, pp. 490-501, Jan. 2012.

[26] L. C. Van Atta, "Electromagnetic reflector," U.S.A. Patent 2908002 , Oct. 6, 1959.

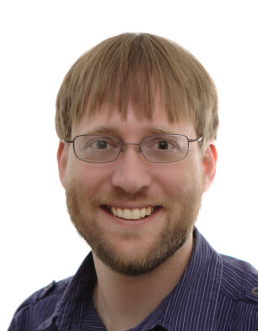

Warren du Plessis (M'00, SM'10) received the B.Eng. (Electronic) and M.Eng. (Electronic) and $\mathrm{Ph} . \mathrm{D}$. (Engineering) degrees from the University of Pretoria in 1998, 2003 and 2010 respectively, winning numerous academic awards including the prestigious ViceChancellor and Principal's Medal.

He spent two years as a lecturer at the University of Pretoria, and then joined Grintek Antennas as a design engineer for almost four years, followed by six years at the Council for Scientific and Industrial Research (CSIR) $\mathrm{He}$ is currently an Associate Professor at the University of Pretoria, and his primary research interests are cross-eye jamming and thinned antenna arrays. 